\title{
ASPECTOS ANATÔMICOS DO RIM DE BÚFALOS
}

\author{
Érica Lieberg Vianna ${ }^{1}$, Raphael Bernardo Silva Neto², Erivaldo Fernandes Silva ${ }^{2}$, \\ José Rômulo Soares dos Santos², Rosa Helena Soares Ferraz ${ }^{3}$, Danilo José Ayres \\ de Menezes $^{2}$
}

\author{
1 UNIMES \\ 2 UFCG \\ 3 UFMT \\ Correspondência: Danilo José Ayres de Menezes: mdanayres@gmail.com
}

RESUMO: A baixa disponibilidade de informações sobre a morfofisiologia de búfalos é um fator limitante para o desenvolvimento da produção desses animais. Nesse sentido, os rins, por serem importantes na manutenção da homeostase do organismo, merecem particular atenção. Assim, este trabalho objetivou estudar a anatomia e a segmentação de rins de búfalo, fornecendo dados para o conhecimento anátomo cirúrgico da espécie. Para tanto, foram coletados 14 pares de rins de búfalos cujos vasos arteriais e venosos foram preenchidos, respectivamente, com acetato de vinil corado com pigmento vermelho e gelatina comercial incolor corada com tinta nanquim azul. Após a disseção, descreveu-se a anatomia externa do órgão e a distribuição dos vasos sanguíneos na região hilar do mesmo. O rim direito de búfalo apresenta forma oval e o esquerdo torcido em seu eixo longitudinal. A superfície dividida por fissuras forma lobos renais, sendo a variação para os rins direito e esquerdo, respectivamente, de 20 à $38(29,57 \pm 4,19)$ e de 21 à $38(30,64 \pm 3,85)$. Tanto a vascularização arterial quanto venosa apresentou variação no número de ramos hilares, tendo mostrado uma média de 10 artérias e 21 veias. Concluiu-se que os rins do búfalo apresentam características anatômicas semelhantes ao observado no bovino, porém, com diferenças que permitem a identificação da espécie, como o rim direito menos alongado e as fissuras que dividem os lobos não tão profundas quando comparado aos rins de bovino.

Palavras-chave: búfalos; morfologia renal; vascularização

\section{ANATOMIC ASPECTS OF THE KIDNEY OF BUFFALOES}

\begin{abstract}
The shortage of information on buffalo morphology and physiology is a limiting factor for the development of the production of these animals. The kidney deserves special attention for its importance on maintaining the organism homeostasis. Thus, the aim of the present study was to research the vascular segmentation and kidney morphology of the buffalo, supplying data for anatomical and surgical knowledge about this specie. Fifteen pairs of kidneys were collected, arterial a venous vessels were injected, respectively, with red pigment stained vinyl and colorless commercial gelatin stained with blue nanking ink. The external anatomy of the organ and distribution of the kidney arteries and veins at hilus were described. The right kidney was oval in shape and left kidney twisted on its longitudinal axis. The surfaces of the buffalo kidneys was divided by fissures from 20 to 38 $(29,57 \pm 4,19)$ and 21 to $38(30,64 \pm 3,85)$ lobes, respectively to right and left kidney. Both the arterial and venous vascularization presented variation in the number of branches and showed a mean of 10 arteries and 21 veins hilares. It was concluded that the kidneys of the buffalo presented anatomic characteristics similar to those observed in bovines but with differences that permit identification of the species, such as the less elongated right kidney and the fissures that divide the lobes that were not as deep compared to those of bovine kidneys.
\end{abstract}

Key Words: buffaloes ; kidney morphology; vascularization 


\section{INTRODUÇÃO}

A pecuária mundial desperta crescente interesse pela bubalinocultura. Esta atividade é uma fonte alternativa viável na produção de proteína de origem animal de alto valor biológico e de forma econômica. Tratase de animais de grande rusticidade, associado a sua capacidade de adaptação as adversidades do meio ambiente, com precocidade, longevidade, vida útil produtiva e reprodutiva de 15 a 20 anos, taxa de natalidade superior a $80 \%$ e de mortalidade inferior a $3 \%$ ao ano (MOREIRA et al., 1994).

Os búfalos domésticos (Bubalus bubalis Linnaeus, 1758) pertencem a ordem Artiodactyla, família Bovidae e subfamília Bovinae, que é composta por cinco espécies (ITIS, 2014). No Brasil, quatro raças bubalinas são reconhecidas: Mediterrânea, Murrah, Jafarabadi e Carabao. Os animais da raça Mediterrâneo, conhecidos como búfalo preto ou italiano, possuem aptidão tanto para produção de carne quanto de leite, têm porte médio e são medianamente compactos (ABCB, 2013).

A baixa disponibilidade de informações sobre a morfofisiologia destes animais é um fator limitante para os estudos na área de produção animal, particularmente aqueles relativos a anatomia, pois são fundamentais para subsidiar pesquisas aplicadas, ou mesmo para estabelecer o padrão da espécie bem como as características raciais.

Por seu papel indispensável na fisiologia animal, os órgãos urinários merecem papel de destaque, sendo os rins indubitavelmente relevantes na manutenção da homeostase, pela remoção de produtos finais do metabolismo e excreção de metabólitos do sangue por meio da filtração do plasma e retenção de substâncias necessárias ao organismo, como proteínas, água e eletrólitos (CUNNINGHAM, 2008; FEITOSA, 2008). Por outro lado, considerando o subsídio que a anatomia oferece para a terapêutica cirúrgica, a expressão segmento anatomocirúrgico foi inicialmente utilizada por Didio (1999), segundo o qual, em cada rim é possível identificar segmentos, que se baseiam na distribuição independente de artérias e veias, permitindo nefrectomia parcial na tentativa de conservar o parênquima renal saudável. Dessa forma, a segmentação arterial e venosa vem sendo investigado em diferentes espécies para fundamentação cirúrgica e compreensão desse padrão entre os diferentes grupos animais. A vascularização arterial do rim é bastante variada de acordo com as espécies de animais domésticos (NICKEL et al., 1979; GETTY, 1986; DYCE, 2010; KÖNING E LIEBICH, 2011). Em bovinos, observa-se que a distribuição dos ramos arteriais é resultante do tronco renal que varia entre os indivíduos. Essas diferenças anatômicas não são somente interespecíficas, mas também estão sujeitas as variações entre as diferentes raças de uma mesma espécie (BLAVIGNAC,1964; TEIXEIRA FILHO, 1982; MARIANA e BORELLI, 1988), sendo no búfalo descrito para a raça Murrah (Miglino et al., 1987).

Este trabalho objetivou fornecer dados para 0 aprimoramento do conhecimento sobre a anatomia e o padrão de segmentação vascular dos rins de búfalo da raça Mediterrânea como subsídio para pesquisas aplicadas nas diversas áreas de conhecimento que permeiam a criação da espécie.

\section{MATERIAL E MÉTODOS}

No abatedouro da Fazenda São Pedro, município de Itanhaém/SP, quatorze pares de rins de búfalos da 
raça Mediterrânea foram coletados após evisceração. Os rins foram retirados da carcaça aos pares, com respectivos segmentos da aorta abdominal e veia cava caudal e foram identificados quanto a antimeria, conforme observação da disposição vascular e uretral, sendo a artéria dorsal , a veia ventral e o ureter caudal , observada antes da retirada dos órgão da carcaça. Em sequência, os rins foram dissecados para a retirada da cápsula adiposa, necessário para a descrição de sua forma e quantificação da lobação. Próximo a aorta e a veia cava caudal, respectivamente, a artéria e a veia renal foram dissecadas e canulados para a realização da técnica de preenchimento vascular com acetato de vinil corado com pigmento vermelho (artéria) e gelatina comercial incolor corada com tinta nanquim azul (veia). Após resfriamento a $8^{\circ} \mathrm{C}$ por 24 horas, os rins foram imersos em solução de ácido sulfúrico a $30 \%$ para corrosão do tecido renal e evidenciação do molde vascular arterial e venoso. O número e o padrão de distribuição desses vasos sanguíneos na região hilar foram caracterizados antes e depois do processo de corrosão. Para a esquematização do padrão de distribuição dos vasos sanguíneos, os rins foram mapeados em quatro setores: crâniodorsal, crânioventral, caudodorsal e caudoventral. A representação dos ramos arteriais e das raízes venosas foram realizadas na região hilar dos rins após a rotação dos mesmos em $90^{\circ}$ no sentido mediolateral.

\section{RESULTADOS E DISCUSSÃO}

Os rins de búfalo (Figura 1) da raça Mediterrânea são mais largos e menos alongados quando comparados aos de bovino (GETTY, 1986). A diferença entre a forma dos rins direito $e$ esquerdo relatado para a espécie bovina (NICKEL et al., 1979; GETTY,
1986; DYCE, 2010) também é observada em búfalo, sendo o rim direito oval irregular, achatado dorsoventralmente enquanto o esquerdo é torcido em seu eixo longitudinal, com a porção caudal mais larga e arredondada e a cranial mais estreita. A superfície dos rins, de coloração marrom-avermelhada, apresenta sulcos de diferentes profundidades que delimitam lobos de vários tamanhos assemelhando-se ao observado em bovinos (SCHWARZE e SCHROEDER, 1970; NICKEL et al.,1979; GETTY, 1986; FRANDSON, 2010; KÖNING e LIEBICH, 2011). Os lobos renais poligonais, cujas bases periféricas largas estão delimitadas pelos sulcos, se estreitam em direção à pelve, característica também verificada em bovinos (DOBBERSTEIN

e HOFFMANN, 1964; NUSSHAG, 1967; GETTY, 1986; FRANDSON, 2010; KÖNING e LIEBICH, 2011).

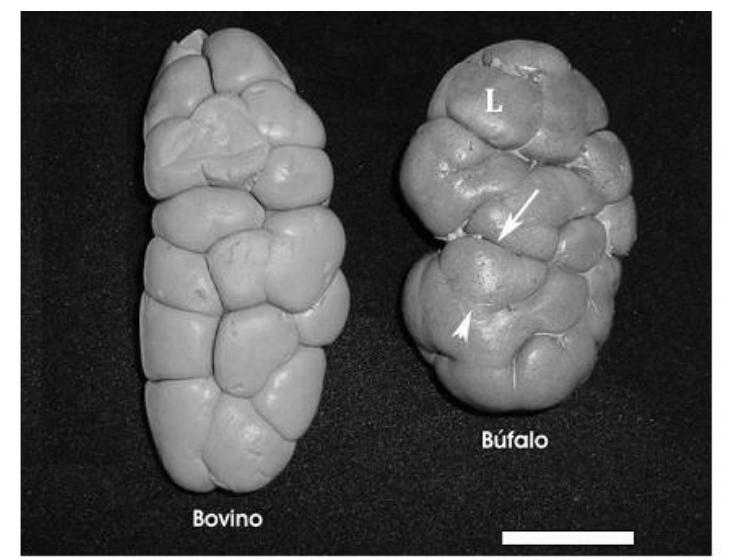

Figura 1 - Vista dorsal de rim direito de bovino e de búf alo. Observar a diferença de forma entre as duas espécies e padrão de lobação cujos lobos do rim de búfalo $(L)$ são delimitados por sulcos profundos (seta) e superficiais (cabeça de seta; Barra $=3 \mathrm{~cm}$ ).

No entanto, os sulcos que delimitam os lobos renais em búfalos podem apresentar diferentes profundidades, o que evidencia uma tendência para a fusão da base desses lobos onde dois ou mais lobos formam lobo único (Figura 1), o que não é observado no bovino (SCHWARZE e SCHROEDER, 1970; FRANDSON, 2010; DYCE, 2010; KÖNING e 
LIEBICH, 2011). A fusão dos lobos renais do búfalo ocorre desde a zona superficial da substância cortical até a parte periférica da área interna da substância medular, porém, nos bovinos estes sulcos são mais profundos e a união dos lobos ocorre apenas na zona corticomedular SCHROEDER, 1970).

O número de lobos nos rins direito e esquerdo (Tabela 1) variam, respectivamente, de 20 à $38(29,57 \pm$ 4,19 lobos) e de 21 à $38(30,64 \pm 3,85$ lobos), característica também observada em bovino (GETTY, 1986), que apresenta aproximadamente de 17 a 24 lobos renais, segundo Ellenberger e Baum (1932), 18 a 22 de acordo com Bruni e Zimmerl (1947) e de 18 a 25 segundo Nickel et al. (1979). Os valores aproximados para os bovinos citados por diferentes autores possivelmente decorram de variações raciais, sendo evidente o maior número de lobos observados em búfalos, embora tenha sido demonstrando a tendência para a fusão dos mesmos.

Tabela 1 - Número de lobos observados extemamente nos rins direito e esquerdo de bưfalos.

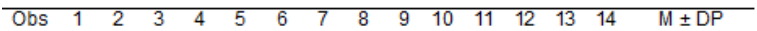

\begin{tabular}{llllllllllllllll}
\hline RD & 33 & 20 & 22 & 24 & 32 & 24 & 28 & 35 & 32 & 30 & 32 & 28 & 36 & 38 & $29,57 \pm 5,26$
\end{tabular}

$\begin{array}{llllllllllllllll}\mathrm{RE} & 32 & 21 & 26 & 27 & 34 & 27 & 26 & 38 & 33 & 28 & 34 & 32 & 33 & 38 & 30,64 \pm 4,74\end{array}$

Obs - Número do rim observado; $\mathrm{RD}$ - Rim Direito; RE - Rim Esquerdo; $\mathrm{M} \pm \mathrm{DP}$ Média \pm Desvio Padrão.

$\mathrm{Na}$ distribuição dos vasos arteriais e venosos observa-se que os vasos arteriais penetram nos rins no centro da região hilar, enquanto que os vasos venosos emergem a partir da região periférica do órgão (Figura 2).

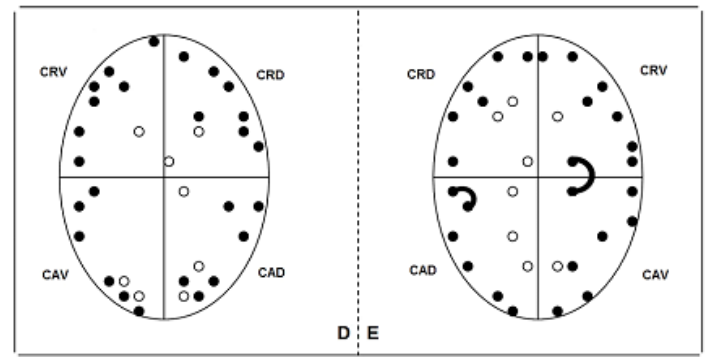

Figura 2 - Representação esquemática dos ramos arteriais (?) e das raizes venosas (?) na região hilar nos rins direito (D) e esquerdo (E) em búfalos da raça caudodorsal; CAV: caudoventral.
Quanto ao número de ramos arteriais (Tabela 2) nos búfalos da raça Mediterrânea a artéria renal ao penetrar na região hilar dos rins direito $e$ esquerdo divide-se, respectivamente, em sete a quatorze (predominância de oito) e em cinco a quatorze ramos arteriais (predominância de 11), semelhante aos resultados obtidos por Miglino et al. (1987) em búfalos da raça Murrah, onde foi relatado para os rins direito e esquerdo, respectivamente, a presença de cinco a dezessete (predominância de onze) e de sete a dezenove ramos arteriais (predominância de onze). Em apenas uma amostra, a artéria renal divide-se em seis e cinco ramos, respectivamente, para o rim direito $e$ esquerdo, padrão esse semelhante ao de bovinos citado por Blavignac (1964) que relatou a presença de três a sete ramos; Schwarze e Schroeder (1972), cinco a oito ramos e Mariana e Borelli (1988), para bovinos da raça nelore, três a nove ramos arteriais (prevalência de quatro).

\begin{tabular}{|c|c|c|c|c|}
\hline \multirow[b]{2}{*}{ RIM } & \multicolumn{2}{|c|}{ RAMOS ARTE RIAIS } & \multicolumn{2}{|c|}{ RAMOS VENOSOS } \\
\hline & $\begin{array}{l}\mathrm{N}^{\circ} \mathrm{de} \\
\text { Vasos }\end{array}$ & $\begin{array}{l}\text { F requência } \\
\text { Relativa (\%) }\end{array}$ & $\begin{array}{l}N^{\circ} \text { de } \\
\text { Vasos }\end{array}$ & $\begin{array}{l}\text { F requên cia } \\
\text { Relativa }(\%)\end{array}$ \\
\hline Direito & \begin{tabular}{|c|}
7 \\
8 \\
9 \\
10 \\
11 \\
12 \\
14 \\
14
\end{tabular} & $\begin{array}{c}7,17 \\
28,56 \\
21,42 \\
14,28 \\
7,14 \\
7,14 \\
14,28\end{array}$ & $\begin{array}{l}13 \\
14 \\
17 \\
19 \\
21 \\
23 \\
24 \\
25 \\
26 \\
27 \\
27\end{array}$ & $\begin{array}{c}7,14 \\
7,14 \\
7,14 \\
7,14 \\
14,28 \\
14,28 \\
7,14 \\
7,14 \\
21,42 \\
7,14\end{array}$ \\
\hline MEDIA & 10 & & 21 & \\
\hline Esquerdo & $\begin{array}{c}5 \\
7 \\
8 \\
9 \\
10 \\
11 \\
13 \\
14\end{array}$ & $\begin{array}{c}14,28 \\
7,14 \\
7,14 \\
14,28 \\
14,28 \\
21,42 \\
14,28 \\
7,14\end{array}$ & $\begin{array}{l}14 \\
16 \\
18 \\
19 \\
22 \\
23 \\
24 \\
26 \\
26 \\
27\end{array}$ & $\begin{array}{c}14,28 \\
7,14 \\
21,42 \\
7,14 \\
7,14 \\
21,42 \\
7,14 \\
7,14 \\
7,14\end{array}$ \\
\hline MEDIA & 10 & & 21 & \\
\hline
\end{tabular}

Quanto à vascularização venosa (Tabela 2), em todas as amostras, os rins direito e esquerdo apresentaram apenas uma veia renal drenando para a veia cava caudal, conforme descrito para bovinos pelos tratadistas Dobberstein e Hoffmann (1964), 
Schwarze e Schroeder (1972) e Nickel et al. (1979).

A formação de veia renal resulta da confluência das raízes venosas sendo observado para os rins direito e esquerdo de búfalos da raça Mediterrânea, respectivamente, treze a vinte e sete e quatorze a vinte e sete raízes venosas. Esses valores foram superiores aos descritos por Miglino et al. (1987) para búfalos da raça Murrah, onde as raízes venosas na região hilar dos rins direito e esquerdo foram, respectivamente, de sete a quatorze (maior frequência de onze) e de seis a vinte e duas (maior frequência de dez). O número de vasos venosos renais em bovinos também foi menor, semelhante ao observado na raça Murrah. Assim, segundo Teixeira Filho et al. (1996), em bovinos da raça Hereford, as veias apresentam quatro a doze (maior frequência de oito) e três a dez (mais frequentemente seis) ramos venosos, respectivamente, para os rins direito $\mathrm{e}$ esquerdo e Mariana e Borelli (1988) identificaram em bovinos da raça Nelore variação de duas a dez raízes hilares(maior frequência de cinco) para ambos os rins.

Em apenas um rim esquerdo foi observado duas anastomoses onde uma emitiu um ramo do setor caudoventral para o setor crânioventral e outra dentro do mesmo setor caudodorsal (Figura 2).

\section{CONCLUSÃO}

Os rins do búfalo da raça

Mediterrânea

apresentam

características anatômicas semelhantes ao observado no bovino , porém, com diferenças que permitam a identificação da espécie, como o rim direito menos alongado e as fissuras que dividem os lobos não tão bem definidos quando comparado aos rins do bovino. Em ambos os rins, tanto a vascularização arterial quanto a venosa apresentam variação quanto ao número de ramos hílares, sendo a maior variação para os ramos venosos.

\section{AGRADECIMENTOS}

A Universidade Metropolitana de Santos-UNIMES, pelo apoio financeiro a este trabalho. Aos professores Dr. Vicente Borelli da Universidade Paulista, São Paulo, SP, Brasil e Dra. Maria Acelina Martins de Carvalho da Universidade Federal do Piauí, pela valiosa ajuda na sugestão dos procedimentos metodológicos do trabalho.

\section{REFERÊNCIAS}

ASSOCIAÇÃO BRASILEIRA DE CRIADORES

DE BÚFALOS. Site oficial. Disponível em: <http://www.bufalo.com.br/racas.html> Acesso em: 28/08/2013.

\section{BLAVIGNAC, B. Recherches sur la} vascularisation et l'innervation des reinschez le boeuf. Lyon, 1964. 54p. Tese de Doutorado EscolaNationaleVétérinaire de Lyon.

CUNNINGHAM, J. G. Tratado de fisiologia veterinária 4aa ed. Rio de Janeiro: Editora Elsevier, 2008.

DIDIO, L. J.A. Sistema Urinário. In:

Tratado de Anatomia Aplicada. Vol. 2. São Paulo, Brasil: Pólus Editorial, 1999. Cap.15, p.604-612.

DOBBERSTEIN, J. \& HOFFMANN, G. Lehrbuch der vergleichenden Anatomie der Haustiere.Leipzig, S. Hirzel, v.3, p.50, 1964.

DYCE, K. M. et al.; O Abdome dos Ruminantes. In:__ Tratado de Anatomia Veterinária.

4ㄹ. ed. Rio de Janeiro:Elsevier, 2010. Cap.28, p.696- 697 .

FRANDSON, R.D.; WILKE W.L. FAILS, A. .D.; O Sistema Urinário. In: Anatomia e

Fisiologia dos Animais de Fazenda. 6ª ed. Rio de Janeiro: Guanabara Koogan, 2010. Cap.23, p.338.

FEITOSA, F.L.F. Semiologia Veterinária: a arte do diagnóstico. $2^{\mathrm{a}}$ ed. Editora Roca, São Paulo. 2008. Cap.10, p.428

GETTY, Robert; Aparelho Urogenital do Ruminante. In: Anatomia dos Animais 
Domésticos.1v. ed.5. Rio de Janeiro: Guanabara Koogan, 1986. Cap.31. p.879-881

KÖNING, H. E.; LIEBICH, H.G. Órgãos Urinários In: Anatomia dos animais

Domésticos Texto e Atlas Colorido. $4^{\mathrm{a}}$ ed. Porto Alegre: Editora Artmed, 2011. Cap.9, p. $411-418$.

MARIANA, A. N. B. \& BORELLI, V. Contribuição ao estudo dos elementos vasculares, arteriais e venosos, do hilo renal, em bovinos da raça Nelore. Revista da Faculdade de Medicina Veterinária e Zootecnia da Universidade de São Paulo, v.25. p.53-70, 1988.

MIGLINO M. A.; DIDIO L. J.; BORELLI, V. Contribuição ao estudo dos elementos vasculares, arteriais e venosos na região hilar do rim em búfalos da raça Murrah.

Comunicações Científicas da Faculdade de Medicina Veterinária e Zootecnia da Universidade de São Paulo, v.11, n.2, p.142-3, 1987.

MOREIRA, P.; COSTA, A. L.; VALENTIN, J. F. Comportamento produtivo e reprodutivo de bubalinos mestiços Murrah-Mediterrâneo em pastagem cultivada em terra firme no Estado do Acre. Rio Branco: Emprapa-CPAF-Acre, 1994. p. 19

NICKEL, R.; SCHUMMER, A.; SEIFERLE, E.; SACK, W. O. The viscera of the domestic mammals. Berlin, Paul Parey, 1979. p.287-296.

NUSSHAG, W. Compendio de Anatomia y Fisiologia de los Animales Domésticos.

Espanha, Editorial Acribia, 1967. p. 161-162.

PEREIRA, F.T.V. Desenvolvimento do placentônio em búfalos (BubalusBubalis.

Faculdade de Medicina Veterinária e Zootecnia - Universidade de São Paulo. São Paulo, 2000.

SCHWARZE, E. \& SCHROEDER, L.

Compendio de anatomia veterinaria.

Zaragoza, Acribia, 1970. v.2 - el sistema visceral, p.231.

SCHWARZE, E. \& SCHROEDER, L.

Compendio de anatomia veterinária.

Zaragoza, Acribia,. v.3, p.72-98, 1972.

TEIXEIRA FILHO, A. Contribuição ao estudo dos elementos vasculares, arteriais e venosos, do hilo renal, em bovinos da raça Hereford. São Paulo, 1982. 59 p. Dissertação (Mestrado) - Faculdade de Medicina Veterinária e Zootecnia da Universidade de São Paulo. 